\section{Relación entre el colesterol de HDL, los niveles muy bajos de colesterol de LDL y los episodios cardiovasculares agudos graves}

Los estudios de población han demostrado de manera consistente que el nivel bajo de colesterol de lipoproteínas de alta densidad (HDL) es un factor predictivo independiente de enfermedades cardiovasculares. El análisis de los resultados de cuatro grandes estudios demuestran que por cada incremento de $1 \mathrm{mg} / \mathrm{dL}(0,03 \mathrm{mmol} / \mathrm{L})$ de colesterol de HDL disminuye el riesgo de enfermedad coronaria en $2-3 \%$. Por otra parte, el uso de estatinas para reducir los niveles de colesterol de lipoproteínas de baja densidad (LDL) ha permitido reducir considerablemente los problemas cardiovasculares en varios grupos de riesgo. Por cada $40 \mathrm{mg} / \mathrm{dL}$ $(1,0 \mathrm{mmol} / \mathrm{L})$ de reducción de los niveles de colesterol de LDL, disminuye el riesgo de enfermedades cardiovasculares en $24 \%$.

Estos hallazgos respaldan la idea de que el aumento de los niveles de colesterol de HDL debe ser un objetivo terapéutico y de salud pública adicional e independiente de la reducción de los niveles de colesterol de LDL. Sin embargo, no está claro el valor que pueden tener los niveles de colesterol de HDL cuando el colesterol de LDL se encuentra en niveles muy bajos.

Para aclarar este punto se hizo un análisis post hoc de los datos del ensayo Treating to New Targets (TNT), recientemente concluido. El objetivo fue analizar la relación existente entre la frecuencia de episodios cardiovasculares agudos graves y los niveles de colesterol de HDL en una población de pacientes con signos clínicos de enfermedad cardíaca coronaria que habían recibido tratamiento con estatinas. También se analizó si esa posible relación se mantenía cuando los niveles de colesterol de LDL se encontraban por debajo de $70 \mathrm{mg} / \mathrm{dL}$. Para ello se estratificaron en quintiles los 9770 participantes del estudio TNT que contaban con los datos de colesterol de HDL, según el nivel de colesterol de
HDL al tercer mes de la fase de tratamiento con doble enmascaramiento.

Se observó que el nivel de colesterol de HDL en los pacientes que tomaron estatinas era un factor predictivo de la ocurrencia de episodios cardiovasculares agudos graves, tanto cuando se tomó el colesterol de HDL como una variable continua como cuando se analizaron los pacientes según los quintiles de niveles de colesterol de HDL, aunque en este último caso el nivel de significación fue bajo $(P=0,05)$. Incluso en el grupo de pacientes con niveles de colesterol de LDL por debajo de $70 \mathrm{mg} / \mathrm{dL}$, los pacientes ubicados en el quintil más alto (mayores niveles de colesterol de HDL) presentaron menor riesgo de ese tipo de episodios que los que estaban en el quintil más bajo $(P=0,03)$.

Según este estudio, el nivel de colesterol de HDL permaneció siendo un factor predictivo de episodios cardiovasculares agudos graves en la cohorte de estudio, aun después de tomar en cuenta todos los otros factores de riesgo, en particular los niveles de colesterol de LDL. Este efecto fue más pronunciado cuando los niveles de colesterol de HDL se tomaron como variable continua que cuando se analizaron por quintiles según el nivel de colesterol de LDL a los 3 meses de tratamiento con estatinas. Estos resultados indican que los niveles atípicos de colesterol de HDL en sí pueden desempeñar un papel importante en la relación observada.

El hecho de que después de ajustar por las covariables, el valor predictivo del nivel de colesterol de HDL fuera bajo, confirma la hipótesis de que en los pacientes con enfermedad coronaria, los niveles altos de colesterol de HDL pueden contrarrestar el aumento del riesgo provocado por los niveles altos de colesterol de LDL. Incluso en el grupo con los niveles más bajos de colesterol de LDL, se redujo el riesgo de episodios cardiovasculares agudos graves en los pacientes con niveles de colesterol de HDL más elevados en comparación con los que tenían los niveles más bajos.

Debido a que según este análisis, tanto los niveles de colesterol de HDL como los de LDL eran factores predictivos independientes de episodios cardiovasculares agudos graves en todos los niveles de colesterol de HDL y de LDL, era de esperar que la razón entre ambos tipos de colesterol tuviera también valor predictivo para ese tipo de episodios. Se observó también un resultado similar con relación a la razón entre el colesterol total y el colesterol de HDL.

En resumen, este análisis secundario de los datos del estudio TNT demostró que los niveles de colesterol de HDL en pacientes con diagnóstico clínico de enfermedad coronaria que recibían tratamiento con estatina constituyen un factor predictivo inverso independiente de futuros episodios 
cardiovasculares agudos graves. Cuando se tomó en cuenta el nivel de colesterol de LDL alcanzado por los pacientes bajo tratamiento, el papel desempeñado por el colesterol de HDL fue menos marcado, aunque mantuvo su significación. Esta relación se mantuvo significativa incluso en pacientes con niveles de colesterol de LDL por debajo de $70 \mathrm{mg} / \mathrm{dL}$. (Barter P, Gotto AM, LaRosa JC, Maroni J, Szarek M, Grundy SM, et al. HDL cholesterol, very low levels of LDL cholesterol, and cardiovascular events. N Engl J Med. 2007;357:1301-10.) 